\title{
Evaluación del proyecto de Sistematización e Informatización del Inventario de Yacimientos Arqueológicos de Andalucía
}

\author{
Silvia Fernández Cacho \\ Pilar Modéjar Fdez. de Quincoces \\ José Manuel Díaz Iglesias \\ Centro de Documentación IAPH
}

\section{INTRODUCCIÓN}

Durante los años 1996 y 1997 se ha procedido a sistematizar, normalizar e informatizar el Inventario de Yacimientos Arqueológicos de Andalucía (en adelante IYAA). La aplicación informática ARQUEOS, empleada para gestionar la información, se diseñó en 1995 en Access y se adaptó a los campos de información contenidos en la ficha del IYAA elaborada en 1992. No obstante, durante el proceso de informatización se amplió la aplicación para ajustarla a las nuevas necesidades que se detectaban y al empleo de otras fuentes de información.

La finalidad del presente artículo es presentar la evaluación del Proyecto de Sistematización e Informatización del Inventario de Yacimientos Arqueológicos de Andalucía, el proceso seguido y las primeras conclusiones extraídas del mismo para contribuir a la mejor planificación y gestión del Patrimonio Arqueológico de la Comunidad Autónoma de Andalucía que, a través de la Dirección General de Bienes Culturales (en adelante DGBBCC), ha realizado un esfuerzo importante en la elaboración de la Carta Arqueológica de su territorio.

\section{EL IYAA COMO FUENTE DE INFORMACIÓN}

En un trabajo anterior (González-Campos - Fernández, 1996) se expusieron los criterios fundamentales con los cuales se habían diseñado las distintas fichas del IYAA, por lo que solo se realizará un breve comentario sobre las mismas.

\section{I. IYAA 1985-89}

Entre los años 1985 y 1989 puede considerarse que se culmina una primera aproximación a la realidad del Patrimonio Arqueológico Inmueble en Andalucía mediante la realización de un primer Inventario por parte de la DGBBCC. Este Inventario se componía de unas 8.500 fichas, que in- cluía una información genérica de los yacimientos y su localización, expresada en un par de coordenadas UTM, extraídos de una cartografía base a escala 1:50.000.

La información se extrajo inicialmente de la bibliografía existente, requiriéndose en muchos casos una contrastación sobre el terreno para verificarla. Dicha contrastación se planteaba por la necesidad de cualificar una información de cuya fiabilidad se dudaba en muchos casos, además de ser insuficiente para aplicar de forma efectiva una adecuada política de protección ya que la falta de una delimitación precisa, derivada de su localización sobre cartografía base a escala 1:50.000, provocaba retardos importantes en la toma de decisiones.

\subsection{IYAA 1992-1995}

Una vez concluida esta primera aproximación a la realidad del Patrimonio Arqueológico de Andalucía, la Dirección General de Bienes Culturales inició un nuevo proyecto encaminado a mejorar la caracterización y la delimitación de los yacimientos arqueológicos previamente inventariados. Este nuevo proyecto comenzó en el año 1992, seleccionando aquellos yacimientos arqueológicos que necesitaban más urgentemente la revisión de la información disponible, siguiendo los criterios de prioridad marcados por las Delegaciones Provinciales de Cultura. En los años sucesivos se priorizó la actualización de la información arqueológica referida a municipios completos que tenían sus planes de ordenación urbanística en redacción o revisión de manera que la información ya cualificada se incorporara a los mismos para garantizar su protección.

Este objetivo se abordó con el diseño de una nueva ficha más completa que, además de la información relativa a los propios yacimientos arqueológicos, incluía otra más enfocada hacia su gestión, como era la afección de los mismos en los planeamientos tanto urbanos como territoriales, la protección legal con la que contaban, los accesos a las mismas o la ubicación actual de los materiales arqueológicos recuperados.

Del mismo modo, y con la finalidad prioritaria de ejercer una protección más efectiva del Patrimonio Arqueológico, se procedió a la delimitación de los yacimientos arqueológicos sobre una base cartográfica a escala I:10.000, disponible para todo el territorio andaluz, lo 
cual permitía representarlas en forma de polígonos, superando la tradicional ubicación por medio de un punto en una base cartográfica a escala 1:50.000.

\subsection{La gestión de la información}

Durante el periodo comprendido entre los años 1985-1997 dos problemas fundamentales afectan a la gestión de la información del IYAA: la carencia tanto de continuidad en el proceso de revisión, como de un programa informático que automatizara su manipulación.

Por lo que se refiere al primer aspecto, la discontinuidad en el proceso de revisión ralentiza la conclusión de una Carta Arqueológica cualificada, generando problemas derivados de la duplicidad de modelos de ficha. Ello provoca una nueva consecuencia inmediata: la información que ahora se considera revisada y actualizada quedará obsoleta mucho antes de la finalización del proyecto de revisión.

Con respecto al segundo punto, en 1989, como fruto de una colaboración establecida entre la DGBBCC y la Agencia de Medio Ambiente, se procedió a informatizar en tablas de dBASEIII la información contenida en este primer Inventario. Dicha informatización adolecía de graves deficiencias relacionadas, sobre todo, con la normalización de la información.

Por otra parte, la nueva ficha realizada en 1992 estaba diseñada pensando en su ulterior informatización, por lo que ya había incorporado un primer intento de normalización terminológica de los diferentes campos de información y unas normas de cumplimentación más rígidas.

La necesidad de gestionar eficazmente el conjunto de la información diponible en el IYYA con una aplicación informática adecuada a tal efecto condujo al diseño de la Base de Datos del Patrimonio Arqueológico de Andalucía ARQUEOS, a través de una colaboración establecida entre el Centro de Documentación del IAPH y el Servicio de Protección del Patrimonio Histórico de la DGBBCC (GonzálezCampos - Fernández, 1996) que, si bien no ha solucionado los problemas derivados de la falta de continuidad en el proceso de revisión del IYAA, si ha supuesto una mejora considerable en la gestión diaria de la información que contiene.

\section{TRATAMIENTO DE PROBLEMÁTICAS ESPECÍFICAS}

Durante el transcurso de la informatización del IYAA y con la inclusión de nuevas fuentes de información (Catálogo de Yacimientos Arqueológicos con Pinturas Rupestres en Andalucía, Expedientes de Declaración de Bien de Interés Cultural o Inscripción Específica en el CGPHA, Documentación y Análisis del Riesgo Antrópico de los Yacimientos Subacuáticos de Andalucía, etc.),se fueron detectando algunas necesidades de ajuste en la Base de Datos ARQUEOS, tanto a nivel informático como de criterios de normalización que pasan a desglosarse a continuación.

\section{I. Recodificación}

Cuando se diseñó la aplicación ARQUEOS, se incorporó como clave principal un código identificador por cada uno de los registros. Este se compone, al igual que en el IYAA, de tres tramos numéricos: dos dígitos referidos a la provincia, tres al municipio y otros cuatro al yacimiento. A lo largo del proceso de informatización del Inventario los criterios para determinar el valor de este último tramo se han adaptado a las diferentes problemáticas detectadas.

Por un lado, se está trabajando en el diseño de una nueva base de datos que integrará la información de los Bienes Inmuebles Arquitectónicos, Etnológicos y Arqueológicos. Al existir en la actualidad bases de datos independientes por cada una de estas tipologías de Bienes con criterios de codificación semejantes, se ha realizado una recodificación general de todos ellos para evitar duplicidades. Esta recodificación ha incidido en mayor grado en los yacimientos de las provincias de Almería, Cádiz, Córdoba, Granada, Jaén y Málaga.

En el caso de la provincia de Sevilla el primer paso en el proceso de informatización ha sido el cambio de los criterios de codificación de los yacimientos. El código con el que se identificaban los yacimientos de Sevilla en el IYAA, a diferencia del resto de las provincias, consistía en asignar en el último tramo un número secuencial a escala provincial de manera que, para dar de alta un nuevo yacimiento, había que saber cual era el último número asignado en el Inventario a escala provincial. A partir de ahora, el criterio de codificación para Sevilla ha consistido en la adaptación del mismo sistema que utiliza el resto de las provincias andaluzas, asignando un número secuencial en cada ámbito municipal y no provincial.

Por otra parte, se han asignado códigos relacionados a aquellas evidencias arqueológicas que no conforman un yacimiento sino una parte del mismo. Es el caso frecuente de los restos excavados en solares urbanos, que han sido dados de alta individualmente en el Inventario y que ahora, aún teniendo registros independientes en la base de datos, se relacionan a través de un nuevo campo de código relacionado con el código asignado a la ciudad como yacimiento arqueológico. En el caso de los yacimientos dispersos, esta relación se establece basándose en unidades funcionales. A modo de ejemplo, se puede documentar un yacimiento que sea un poblado protohistórico, muy próximo a él su necrópolis, y en el mismo poblado una torre medieval. En este caso los tres están dados de alta con un código independiente pero enlazados por el código relaccionado, que no es otro que el asignado al yacimiento que engloba a las principales unidades funcionales, esto es, el poblado, la torre y la necrópolis. 


\subsection{Yacimientos Subacuáticos}

En la ficha del IYAA de 1992, se contemplaba la posibilidad de discriminar los yacimientos subacuáticos a través de un campo de situación en el medio físico. Dado que el resto de la información quedaba recogida básicamente en los diferentes campos de ARQUEOS, solo se añadieron algunos específicos y se introdujeron nuevos parámetros de búsqueda para adaptar la aplicación a la incorporación de la información de este tipo de yacimientos, recogiendo las sugerencias de los técnicos del Centro de Arqueología Subacuática.

En primer lugar, se ha incorporado un campo que hace referencia al tipo de fondo marino y otro a la visibilidad media del yacimiento, ambos asociados a listados de términos normalizados. Este tipo de información permite planificar el acceso futuro al yacimiento. Por otra parte se añadió un nuevo criterio de selección consistente en la realización de una rutina de búsqueda para consultar o editar únicamente los yacimientos arqueológicos subacuáticos. Este criterio podía combinarse con los ya existentes (código, denominación, localización, clasificación cultural, tipología y nivel de protección) permitiendo seleccionar, por ejemplo, yacimientos subacuáticos con una clasificación cultural y una tipología determinada.

En cuanto a la codificación de los yacimientos subacuáticos hay que señalar que si en el caso de los yacimientos terrestres éste se generaba introduciendo el código de la provincia, del municipio y del yacimiento, en el caso de los subacuáticos dichos límites administrativos desaparecen, por lo que se optó por codificar el yacimiento con respecto a la provincia y el municipio que estuviese mas cerca trazando una línea recta ficticia hasta la costa.

Por último, habría que señalar que mientras la delimitación de los yacimientos en medio terrestre se realiza mediante un polígono de coordenadas UTM, los subacuáticos tradicionalmente se han localizado mediante coordenadas geográficas, al utilizar las cartas naúticas como base cartográfica. Para volcar esta información en ARQUEOS se han convertido dichas coordenadas geográficas a UTM para trabajar con el mismo sistema de proyección. Así mismo apuntar que se han delimitado como polígonos aquellas evidencias arqueológicas que conformaban un yacimiento (pecios, fondeaderos, vertederos...), mientras que los hallazgos puntuales se han localizado mediante un par de coordenadas representadas como un punto en la cartografía.

\subsection{Zonas Arqueológicas}

La inclusión de la información procedente de los Expedientes de Declaración de Bien de Interés Cultural o Inscripción Específica en el CGPHA, ha determinado la inclusión de una serie de campos de información para las Zonas Arqueológicas protegidas. Dichos campos se refieren fundamentalmente a las Instrucciones Particulares, la Justificación de la Delimitación y la Justificación de la Inscripción, así como datos administrativos referidos a la tramitación de los mismos.

\subsection{Normalización terminológica}

Como ya se dijo anteriormente, la Base de Datos ARQUEOS incorporaba desde el principio, asociados a la mayoría de sus campos, listados de términos normalizados, utilizando como base los ya incluidos en las fichas del IYAA y ampliándolos. Posteriormente estos listados se han ido acrecentando conforme avanzaba el proyecto de informatización cuando era necesario o cuando asi lo demandaban los técnicos de las Delegaciones Provinciales que trabajaban en la revisión del mismo.

Actualmente se trabaja en la adaptación de la terminología empleada en la Base de Datos a la normalizada por el Tesauro del Patrimonio Histórico de Andalucía, realizándose tablas de conversión de la información que serán incluídas a corto plazo en la aplicación infórmática. Con ello se ha pretendido agilizar las rutinas de búsqueda por diferentes criterios, la adopción de un lenguaje normalizado para todos los usuarios y la preparación de la aplicación ARQUEOS para el posterior volcado de la información que almacena en la nueva Base de Datos del Patrimonio Histórico de Andalucía.

\subsection{La gestión del IYAA con tecnología SIG}

Con independencia del considerable avance que supone disponer de una Base de Datos para gestionar de forma automática la información del IYAA, la incidencia que estos tienen sobre la ordenación del territorio y la necesidad de disponer de una herramienta de tratamiento espacial de dicha información, hace imprescindible avanzar hacia el diseño de una aplicación de trabajo en soporte SIG.

Si bien este proceso se encuentra todavía en un estado incipiente, el SIPHA contempla este objetivo final por el marcado carácter territorial de mucha de la información que contiene, en base a tres necesidades principales:

- Agilizar y cualificar las actuaciones en materia de protección y evaluación de riesgos que afecten al Patrimonio Histórico de Andalucía.

- Realizar análisis de dinámicas poblacionales y predicciones de zonas de alto potencial arqueológico.

- Elaborar proyectos de Gestión de Recursos Culturales.

Desde el Centro de Documentación del IAPH se están promoviendo diversas iniciativas con carácter experimental. Por una parte, en colaboración con el Instituto de Cartografía de Andalucía, se ha diseñado una primera cobertura Arc-Info de las Zonas Arqueológicas protegidas de Andalucía y se trabaja en coordinación con el Departamento de Prehistoria y Arqueología de la Universidad de Sevilla en la integración de información de la Base de Datos ARQUEOS con el mismo Sistema de Información Geográfica, habiéndose realizado la primera cobertura digital de la totalidad de los yacimientos arqueológicos de Andalucía (Amores et alii, 1997a; 1997b). 
La cobertura se realizó en septiembre de 1996 tomando como base las antiguas tablas .dbf facilitadas por la DGBBCC ya que en la base de datos ARQUEOS sólo se disponía en esos momentos de la información de los yacimientos arqueológicos de la provincia de Huelva. Igualmente se ha adquirido el SIG Arcview 3.0 para gestionar desde el propio Centro de Documentación las coberturas que se van generando y cruzarlas con las provenientes de otros organismos de la Comunidad Autónoma. Ello redundará, a su vez, en la cualificación de la información, ya que la georreferenciación de los yacimientos es deficiente en algunos casos y la detección de este tipo de deficiencias solo es efectiva mediante el uso de la herramienta SIG. A este respecto, se han detectado algunos errores en la asignación de coordenadas y se ha cuantificado (Gráfico I) a nivel provincial el número de yacimientos arqueológicos sin georreferenciar.

La continuación de este trabajo se centra en la actualidad en dos aspectos fundamentales:

- Elaboración de nuevas coberturas basadas en la información disponible en la Base de Datos. En estas nuevas coberturas se representarán los yacimientos como entidades puntuales o poligonales, dependiendo del nivel de detalle de la información que ha servido como fuente de datos. Dichas entidades tendrán asociados al menos el código y la denominación del yacimiento al que representan.

- Conexión mediente consultas SQL de las coberturas gestionadas en Arcview con la totalidad de la in- formación contenida en la base de datos. Dicha conexión se establece a través del código de identificación del yacimiento y su finalidad principal es la elaboración de cartografía temática utilizando como variables cualquiera de los campos normalizados de la base de datos.

\section{ANÁLISIS DE LA INFORMACIÓN}

En el conjunto de la Comunidad Autónoma el nivel de información de los yacimientos arqueológicos es muy dispar, tanto en la cantidad como en la calidad de los datos consignados en las fichas de Inventario.

En la tabla adjunta (Tabla I) se muestra la periodización de las actividades desarrolladas en el marco del Proyecto hasta el momento:

\begin{tabular}{lccc}
\hline & INICIO & FIN & N$^{0}$ Reg. \\
\hline IYAA PROVINCIA DE HUELVA & $01 / 1996$ & $04 / 1996$ & 835 \\
\hline IYAA PROVINCIA DE SEVILLA & $05 / 1996$ & $01 / 1997$ & 2.444 \\
\hline IYAA PROVINCIA DE CÁDIZ & $04 / 1997$ & $06 / 1997$ & 1.124 \\
\hline IYAA PROVINCIA DE CÓRDOBA & $07 / 1997$ & $10 / 1997$ & 1.145 \\
\hline IYAA PROVINCIA DE JAÉN & $04 / 1997$ & $11 / 1997$ & 1.326 \\
\hline IYAA PROVINCIA DE ALMERÍA & $04 / 1997$ & $10 / 1997$ & 1.085 \\
\hline IYAA PROVINCIA DE MÁLAGA & $10 / 1997$ & $11 / 1997$ & 1.122 \\
\hline IYAA PROVINCIA DE GRANADA & $10 / 1997$ & $12 / 1997$ & 372 \\
\hline
\end{tabular}

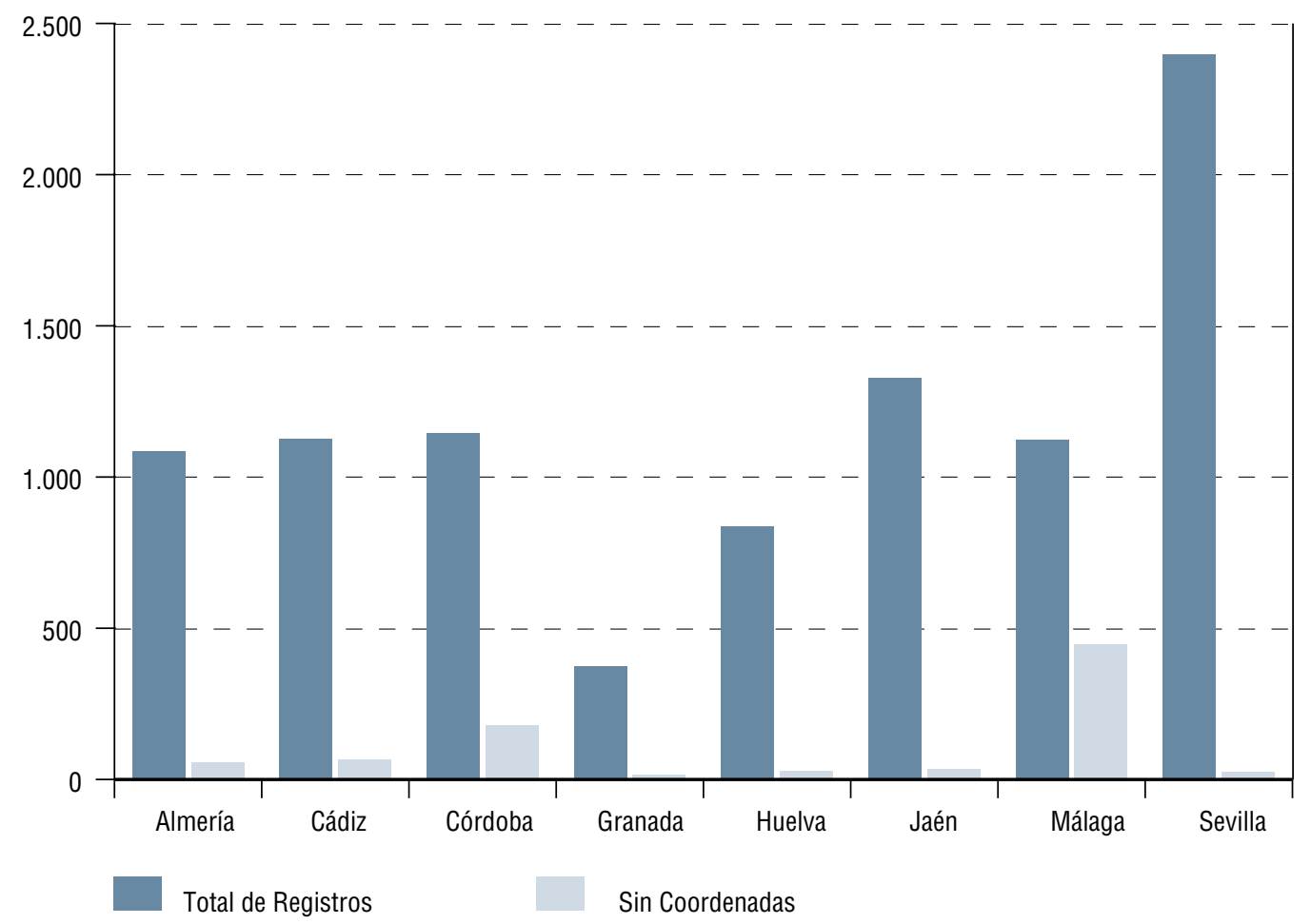




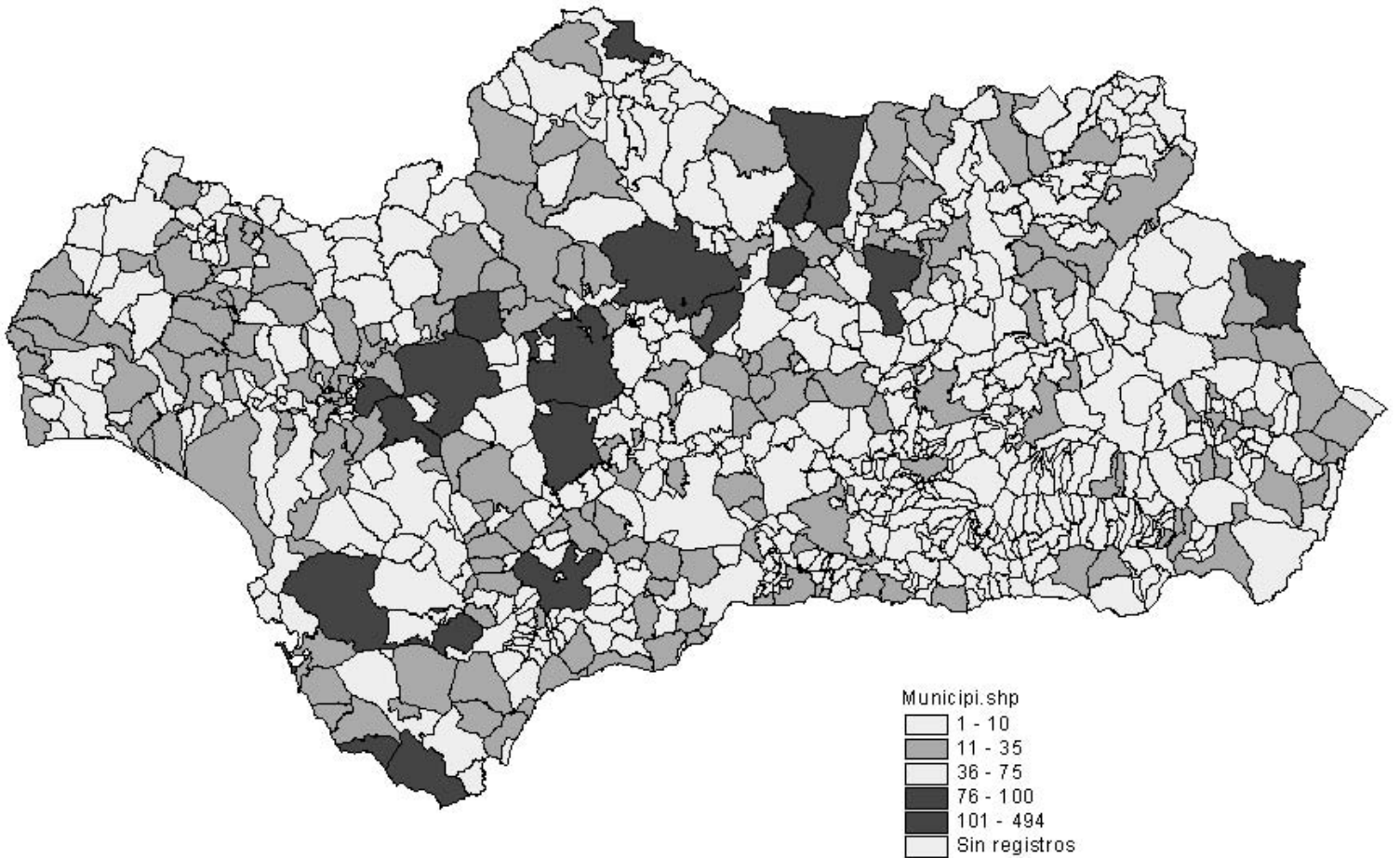

Gráfico 2. CLASIFICACIÓN CULTURAL

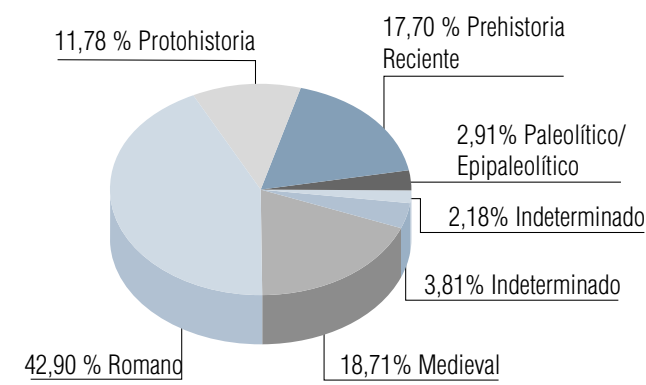

Desde el punto de vista cuantitativo se observa la diversidad de situaciones detectadas a escala provincial en relación con el número de registros existentes en la Base de Datos. A este respecto se detectan una serie de vacíos de información producto (dejando al margen la propia dinamica histórica de ocupación del territorio), en parte, de la falta de actualización del IYAA con los nuevos conocimientos adquiridos tras la realización de actividades arqueológicas y/o la inexistencia de las mismas.

En el mapa I se presenta una clasificación de los municipios de Andalucía en función del número de registros existentes en la Base de Datos, mientras que en el mapa 2 se representa el mismo parámetro en función de la superficie de cada municipio para obtener la densidad real de la información arqueológica disponible para cada uno de ellos.

Excepción hecha de algunos municipios dispersos que se han prospectado de forma más intensiva a través de la realización de cartas arqueológicas específicas, puede observarse que el mayor número de registros se refieren a municipios ubicados a lo largo del Valle del Guadalquivir, más investigado tradicionalmente en comparación con otras áreas.

Se observa claramente los vacios de información en la práctica totalidad de la provincia de Granada, producto del desplazamiento de la investigación universitaria hacia la provincia de Almería, y otras zonas como la Sierra Norte de Sevilla, la Sierra de Cazorla, Segura y las Villas y el tercio sur de la provincia de Jaén, la mitad norte de la provincia de Córdoba y la mitad occidental no costera de la provincia de Málaga.

Promover la investigación en estas zonas a traves de su priorización en el Plan Andaluz de Investigación, permitiría conocer y completar el mapa arqueológico de la Comunidad Autónoma para su mejor tutela.

Un análisis preliminar de la información contenida en la Base de Datos muestra que, en el conjunto de la Comunidad Autónoma, el porcentaje de yacimientos en función de su clasificación cultural (Gráfico 2) es de un $42,90 \%$ asociados, de forma exclusiva o no, a época romana. Le siguen a cierta distancia los medievales $(|8,7| \%)$, los asociados a la prehistoria reciente $(17,70 \%)$ y a la protohistoria (1 1,78\%). En un tercer bloque y en menor número se contablizan los inclui- 

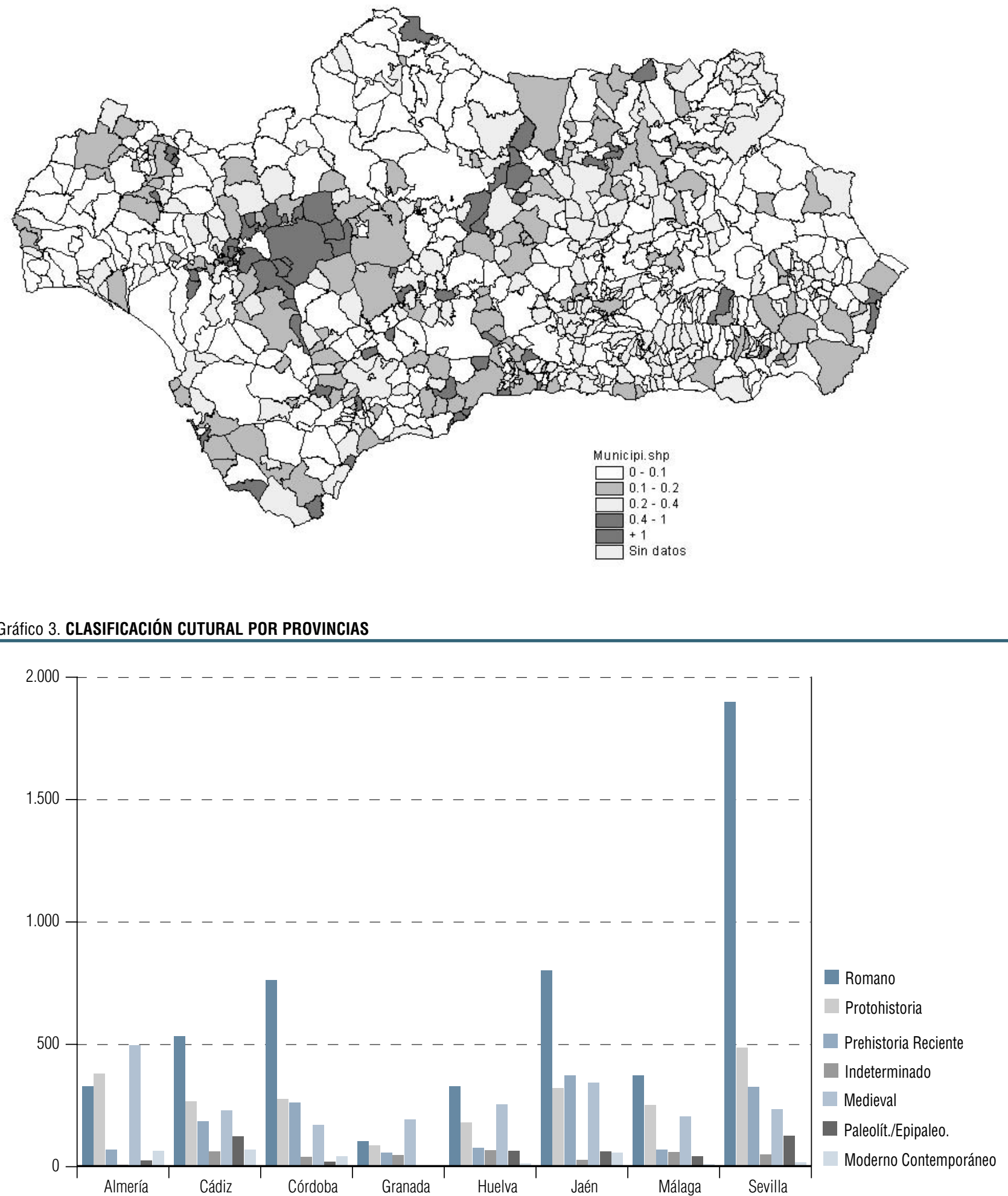

dos en la categoría de moderno/contemporáneo (3,81\%), paleolítico/epipaleolítico (2,91\%) e indeterminados $(2,18 \%)$.

En lineas generales se observa que el número de registros asociados a época romana sobresale del resto en todo el territorio andaluz. Admitiendo esta evidencia, hay que tener presente que una parte considera- ble del inventario se ha nutrido de información bibliográfica, en la cual se reflejaba una orientación de las investigaciones hacia el mundo clásico. Este puede ser el caso de la cuenca media del Guadalquivir. Sin embargo, en provincias como Almería y Granada, donde la investigación ha estado más vinculada a la Prehistoria Reciente, el número de yacimientos de esta época es superior al romano (Gráfico 3). 
Por otra parte, la distribución de los yacimientos en función de su tipología (Gráfico 4) permite observar la existencia de una mayoría que han sido considerados de forma exclusiva o no, como asentamientos (40,4\%), seguidos en menor porcentaje por los lugares de actividad económica (19,1\%) y lugares funerarios ( I4,2\%). Un segundo bloque está formado por yacimientos cuya tipología no ha sido determinada (9,7\%), arquitectura militar (6,4\%), obras y edificios públicos (3,9\%), lugares con representación gráfica $(2,6 \%)$, hallazgos aislados (2,4\%) y lugares cultuales $(0,9 \%)$. Un último bloque englobaria a los yacimientos considerados como vertederos, fondeaderos y pecios $(0,6 \%)$.

Analizando esta información, destacan claramente los registros considerados como asentamientos y lugares de actividad económica ya que son los que presentan más evidencias superficiales, mientras que los lugares funerarios son, generalmente, más dificilmente localizables. La escasa representación de otras tipologías

Gráfico 4. TIPOLOGÍAS

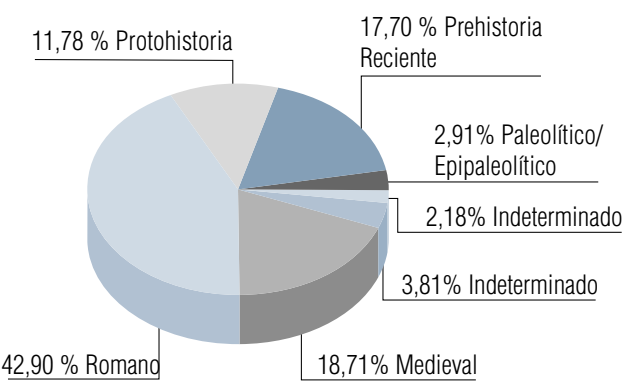

como arquitectura military obras y edificios públicos, puede deberse a su caracter de elementos singulares vinculados a momentos culturales muy determinados.

Por último hay que señalar que la mínima proporción de fondeaderos y pecios tiene su justificación en la pobre tradición de las investigaciones arqueológicas subacuáticas en Andalucía.

Por provincias (Gráfico 5) se puede apuntar el mayor nú- mero de registros asociados a asentamientos con la excepción de la Jaén, donde el número de registros asociados, de forma exclusiva o no, a lugares de actividad económica $(6 / 5)$ supera ligeramente a los asentamientos (565), debido fundamentalmente al elevado número de alfares romanos documentados. Asimismo, el número de registros asociados a lugares funerarios es superior a los lugares de actividad económica en las provincias de Cádiz, Granada y Málaga, siendo menor en el resto de las provincias.

Para finalizar hay que mencionar que del total de yacimientos arqueológicos de Andalucía un 8,2\% de los registros carecen de georreferenciación. El 91,8\% restante se localizan mediante un punto o polígono. Analizando esta información pormenorizadamente se observa que son las provincias de Málaga y Córdoba las que presentan un mayor número de registros sin georreferenciar (39,8\% para el primer caso y 15,5\% para el segundo). El resto de las provincias presentan una proporción más o menos homogénea, destacando del conjunto Sevilla, que siendo la que tiene un mayor número de registros (2.396) solo posee un $1 \%$ de registros sin coordenadas.

\section{LA BASE DE DATOS ARQUEOS Y LA RED DE INFORMACIÓN DEL SIPHA}

\section{I. Implantación en la Dirección General de Bienes Culturales y Delegaciones Provinciales de la Consejería de Cultura}

Desde los inicios del Proyecto de Informatización del Inventario se han mantenido reuniones con los responsa-

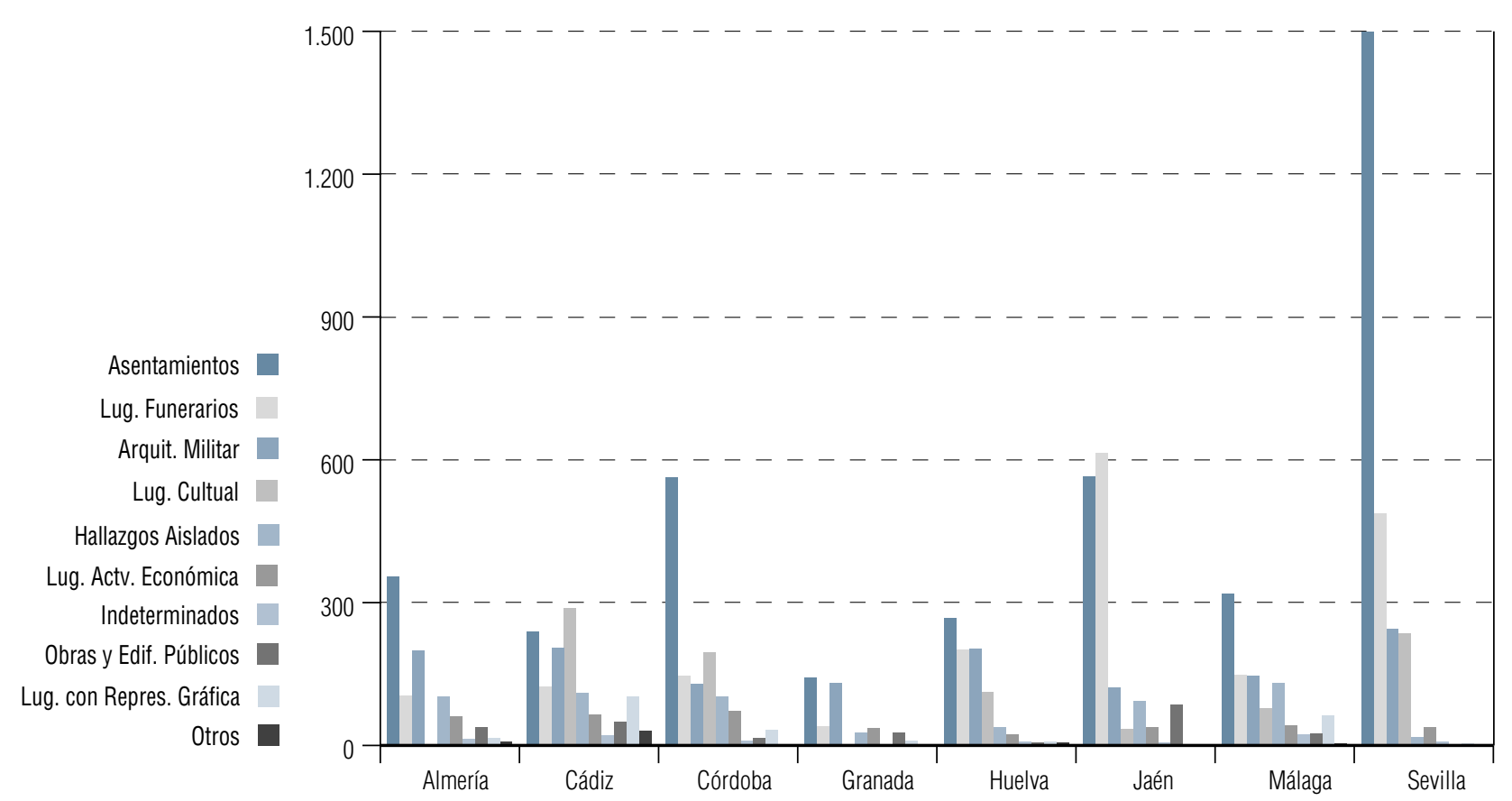


bles de la gestión del IYAA en el Servicio de Protección del PH de la DGBBCC y con los arqueólogos provinciales de las Delegaciones Provinciales de Cultura (en adelante DDPPC), tanto de forma conjunta como bilateral, para informarles de la marcha del proyecto y recibir sus sugerencias. Del mismo modo, conforme se ha ido concluyendo la carga de información del IYAA relativa a cada provincia, se ha enviado a la Delegación correspondiente un informe que incluía un análisis inicial de los datos introducidos en la base de datos, un listado de los yacimientos de la provincia, un ejemplo del informe de salida por impresora de los yacimientos de un municipio concreto y una breve evaluación de las carencias fundamentales detectadas en la información consignada en las fichas, sobre todo las revisadas a partir de 1992

A la expansión informática y de contenidos de la Base de Datos ARQUEOS, durante 1997 le ha seguido su 'expansión institucional'. Efectivamente uno de los objetivos básicos del proyecto es procurar una mejora en la gestión de la información dentro de la administración de cultura (Muñoz, 1998). Avanzando en esa dirección se ha instalado la aplicación completa con toda la información del IYAA y la procedente del resto de fuentes empleadas hasta el momento en la Unidad de Información de la DGBBCC.

Por otra parte, en las DDPPC se han instalado los módulos de consulta y búsqueda con sus correspondientes utilidades (impresión de fichas y listados, búsquedas por diferentes criterios, visualización de datos, etc.) pero sin la posibilidad de manipular la información. En algunos casos puntuales se ha facilitado también el módulo de carga de forma independiente, para abordar proyectos concretos de prospección o revisión de información por parte de la DGBBCC o las DDPPC. Próximamente se instalará este módulo en todas ellas para volcar descentralizadamente la información que se considere necesaria y que deberá ser validada en el Centro de Documentación del IAPH antes de ser cargada definitivamente en la Base de Datos. Con ello se evitará, hasta el momento en que la red informática entre los distintos organismos facilite la labor, que existan tantas Bases de Datos distintas como instituciones que requirieran su uso.

\subsection{Peticiones de Información}

En un primer momento fue el Servicio de Información del Centro de Documentación del IAPH el encargado de dar respuesta a las distintas peticiones de información sobre los yacimientos incluidos en ARQUEOS. Esta función, que se ha desarrollado desde el año 1996, se ha ampliado en Julio de 1997 con la creación de la Unidad de Información en la DGBBCC. El objetivo de ambos servicios es facilitar el acceso a la información del Patrimonio Histórico al usuario que así lo requiera.

Normalmente el tipo de información que se facilita varía en función del usuario y del motivo de la petición. Lo más común es proporcionar un listado de datos básicos sobre los yacimientos en el que figura el código, nombre, provincia, municipio y una clasificación cultural y tipología genérica. Esta información puede ampliarse
Gráfico 6. PETICIONES SEGÚN USUARIOS

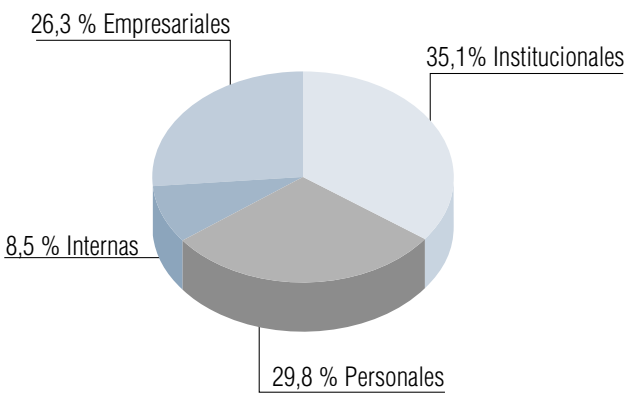

si el usuario lo requiere en función del objetivo de su petición. En este último caso puede facilitarse de forma puntual un modelo de ficha impresa que recoge la totalidad de los datos del yacimiento. En ambos casos la indicación de la delimitación del yacimiento con sus coordenadas queda restringida, proporcionándose sólo en aquellas situaciones en los que la necesidad de las mismas está estrictamente justificada, negándose en todos los casos los datos, referidos a la titularidad de los terrenos donde se ubica.

Analizando las peticiones recibidas, se puede destacar que entre los años 1996 y 1997 se han recibido un total de 97 solicitudes de información de las cuáles 91 han tenido respuesta satisfactoria. En el gráfico adjunto se observa (Gráfico 6) la procedencia de las peticiones agrupadas en cuatro grupos fundamentales: peticiones internas de los distintos centros y profesionales del IAPH (8,5\%), peticiones de instituciones tales como ayuntamientos o departamentos universitarios (35, I \%), un grupo de peticiones personales $(29,8 \%$ ) en las cuáles se engloban diversos profesionales e investigadores no adscritos a una institución concreta y, por último, un grupo de peticiones de empresas que desarrollan estudios de gestión medioambiental o trabajan en el equipamiento de infraestructuras territoriales (26,3\%).

El criterio más común a la hora de realizar las solicitudes de información, consiste en la discriminación de un conjunto de yacimientos en función del ámbito espacial en el que se encuentra, fundamentalmente municipal (69\%). Un segundo grupo de peticiones restringe la petición en función de la tipología, la clasificación cultural o el grado de protección de los yacimientos (13,4\%). Por ultimo, se realizan peticiones de información de yacimientos concretos (7,2\%).

\section{CONCLUSIONES Y PROPUESTAS}

El Servicio de Protección del PH de la DGBBCC ha venido desempeñando sus funciones de tutela del $\mathrm{Pa}-$ trimonio Arqueológico en base a la figura legal del Catálogo General del Patrimonio Histórico de Andalucía, es decir, jurídicamente la Inscripción de una Zona Arqueológica en dicho Catálogo o su declaración como Bien de Interés Cultural es el procedimiento más efi- 
caz de que dispone la administración de Cultura para la protección de la misma.

No obstante, se ha promovido la elaboración de un Inventario de Yacimientos Arqueológicos para prevenir la destrucción de los mismos mediante su inclusión en los catálogos de los planeamientos urbanos y territoriales, - la notificación de su existencia a organismos públicos y privados que, por la naturaleza de sus intervenciones sobre el territorio, demandan este tipo de información.

Como ya se ha comentado anteriormente, para acometer la realización del citado Inventario la DGBBCC diseñó una primera ficha en 1985 que luego fue ampliada en 1992, estableciéndose los campos de información que se consideraron necesarios para desempeñar las competencias de protección que tenían encomendadas.

Por su parte, el Centro de Documentación del IAPH comienza a trabajar en 1995 dentro del marco del Sistema de Información del Patrimonio Histórico de Andalucía en la Informatización del Inventario con dos planteamientos iniciales: por un lado, se realizaba una primera valoración de la documentación existente como punto de partida para su posterior cualificación y acrecentamiento dentro de la estructura marcada por el SIPHA y, por otro, se ofrecía a la DGBBCC una herramienta informática que permitiera la gestión automatizada de la documentación generada en soporte papel.

Finalizada esta primera fase de diseño y carga de información del IYAA en la Base de Datos del Patrimonio Arqueológico de Andalucía, se está comenzando a abordar la segunda fase del proceso de carga de información que trae consigo la ampliación de su módulo de 'Intervenciones y Ubicación de materiales' y la creación de uno nuevo de análisis (mucho más detallado que el actual módulo 'descripción'), para volcar los datos contenidos en la documentación que sobre las intervenciones arqueológicas existe en el Servicio de Investigación de PH de la DGBBCC, las DDPPC y en la Base de Datos de Actividades de Gestión Patrimonial del Centro de Documentación del IAPH. Ello requerirá un estudio previo encaminado a la normalización y sistematización de la información, ya que la documentación no es homogénea para el conjunto de intervenciones, dependiendo del carácter de las mismas (sistemáticas o de urgencia) y, sobre todo, de sus directores. Todo ello supondrá un avance cualitativo -se incluirá una información más detallada de aquellos yacimientos que han sido objeto de actuaciones arqueológicas- y cuantitativo se darán de alta aquellos yacimientos conocidos a través de prospecciones arqueológicas que no han sido incluidos en el IYAA. Ante esto, cabría establecer definitivamente la exigencia a los equipos arqueológicos que realizan prospecciones, ya sea por encargo directo de la administración como en el marco de proyectos de investigación, de cumplimentar la ficha informatizada cuando realicen prospecciones y documenten la existencia de nuevos yacimientos no incluidos en la Base de Datos, o revisarla en el caso de su existencia.

En cuanto a la información del estado de conservación del Patrimonio Arqueológico Inmueble, su siste- matización y carga supondrá un avance esencialmente cualitativo que requerirá una nueva ampliación de la aplicación informática para volcar los datos de las fichas diagnóstico y de los proyectos de conservación y restauración que son competencia del Servicio de Conservación del PH de la DGBBCC.

Por otra parte, la marcha de los diferentes proyectos desarrollados tanto desde el Centro de Documentación como en colaboración con otras instituciones (Mapa Digital de las Aglomeraciones Urbanas, Atlas de Andalucía, Integración de la Base de Datos ARQUEOS y el S.I.G. Arc-Info,...) pueden determinar las prioridades en la revisión de la información de base, procedente del Inventario, o de la recopilada tras la carga de la producida por los Servicios de Investigación y Conservación.

La superación en cuanto al nivel de información de la Base de Datos ARQUEOS respecto al IYAA no invalida la funcionalidad de éste como medio de reconocimiento general del Patrimonio Arqueológico de la Comunidad Autónoma, no siendo operativo cargar excesivamente la ficha manejada para tal fin en la DGBBCC, sobre todo teniendo en cuenta que, para un alto porcentaje de yacimientos, no se dispone de una información adicional a la ya reflejada en la ficha. Así pues, la existencia de una información general homologable a cualquiera de la incluida en otros inventarios nacionales, continúa siendo esencial. Ello no condiciona en absoluto la perentoria necesidad de retomar el proyecto de actualización de la información del IYAA ya que, en un aproximadamente $70 \%$, no está contrastada in situ y, sobre todo, los márgenes de error en su ubicación (I hectárea) derivados de su localización sobre una base cartográfica a escala |:50.000, resultan todavía demasiado elevados.

\section{Bibliografía}

AMORES, F.-HURTADO, V.-MÁRQUEZ, H.-RODRÍGUEZ-BOBADA, M.C.-GARCÍA, L.-LADRÓN DE GUEVARA, M.C.-FERNÁNDEZ, S. 1997a "Planteamientos y Primeros Resultados de la Transferencia a Soporte SIG del Inventario de Yacimientos Arqueológicos de Andalucía". Boletín del Instituto Andaluz del Patrimonio Histórico n 18. Consejería de Cultura de la Junta de Andalucía. Sevilla. Pgs.: I24-I 33. 1997b: "GIS e Patrimonio Archeologico dell'Andalusia: Esperienze preliminari del Centro di Documentazione dell'Istituto Andaluso del Patrimonio Storico". Incontro di Studio Carta Archeologica e Pianificazione Territoriale: un problema politico e metodologico. Roma I0-I 3 Maggio, 1997. Provincia di Roma. Roma. En prensa.

\section{GONZÁLEZ, Y.-FERNÁNDEZ, S}

1996: "La Base de Datos de Zonas Arqueológicas de Andalucía". Boletín del Instituto Andaluz del Patrimonio Histórico n 16. Consejería de Cultura de la Junta de Andalucía. Sevilla. Pgs.: I I 2- I I8.

MUÑOZ, V.

1997: "Las unidades de información del patrimonio histórico andaluz: primeras experiencias" I Jornadas Andaluzas de documentación. Sistemas y Políticas de Información en el Estado de las Autonomías. Situación actual y perspectivas. Sevilla 13-14 de noviembre de 1997. Asociación Andaluza de Documentalistas. Sevilla. Pgs.: 305-310. 